\title{
Predictors of Human Papillomavirus Vaccine uptake or intent among parents of preadolescents and adolescents
}

\author{
Kimberlee Dayal ${ }^{1}$, Sarah Robinson ${ }^{2}$, Jessica Schoening ${ }^{3}$, Mary Catherine Smith ${ }^{4}$, Son Chae Kim ${ }^{* 5}$ \\ ${ }^{1}$ Orthopaedic Specialists of Austin, United States \\ ${ }^{2}$ Concordia University, United States \\ ${ }^{3}$ Austin Area OBGYN \& Fertility, United States \\ ${ }^{4}$ CommUnityCare Federally Qualified Health Centers, United States \\ ${ }^{5}$ St. David's School of Nursing, Texas State University, United States
}

Received: November 19, 2016

Accepted: December 27, $2016 \quad$ Online Published: January 15, 2017

DOI: $10.5430 /$ jnep.v7n6p35

URL: http://dx.doi.org/10.5430/jnep.v7n6p35

\begin{abstract}
Aim: The aim of this study was to examine predictors of human papillomavirus (HPV) vaccine uptake or intent among parents of pre-adolescents and adolescents.

Methods: A cross-sectional descriptive study was conducted among parents of girls aged 9 to 18 years, visiting two primary care clinics in central Texas from September to November 2015. Pearson's product-moment correlation procedures and path analyses based on Health Belief Model were performed.

Results: Path analysis showed that provider recommendation for HPV vaccination $(\beta=0.37 ; p<.001)$ and perceived HPV vaccine harm $(\beta=-0.48 ; p<.001)$ had statistically significant direct effects on HPV vaccine uptake or intent. The perceived HPV vaccine effectiveness was directly influenced by HPV knowledge $(\beta=0.39 ; p<.001)$, empowerment in parent-provider relationships $(\beta=0.30 ; p=.006)$ and parental college education $(\beta=0.23 ; p=.039)$.

Conclusions: Together with parental empowerment fostering an equal partnership with providers, targeted education to improve parental HPV knowledge may convince them of the HPV vaccine effectiveness. This, in turn, may help them put the perceived HPV vaccine harm in proper perspective and allow them to make informed decisions regarding the timely HPV vaccination of their children. Because provider recommendation is one of the most important contributing factors for HPV vaccine uptake or intent, parental education and recommendations from nurses will help reduce the knowledge gaps and empower parents to make the timely decisions to vaccinate their children.
\end{abstract}

Key Words: Human papillomavirus (HPV), Vaccine, Uptake, Intent, Parent-provider relationship

\section{INTRODUCTION}

Human papillomavirus (HPV) infection and HPV-associated cancers are highly preventable with timely vaccination. However, some 14 million new sexually-transmitted HPV infections occur each year and the lifetime disease probabil- ities are greater than $80 \%$ and $90 \%$ for women and men, respectively. ${ }^{[1]}$ Each year, approximately 31,000 new HPVassociated cancers occur among 19,000 women and 12,000 men in the United States, including cervical, oropharyngeal, anal, vulvar, penile, vaginal and rectal cancers. ${ }^{[2]}$ The

*Correspondence: Son Chae Kim; Email: sck30@txstate.edu; Address: St. David's School of Nursing, Texas State University, United States. 
Advisory Committee on Immunization Practices has recommended a series of routine 3-dose HPV vaccinations at target ages of 11 and 12 years for girls since 2007 and for boys since 2011, although the vaccine may be given as early as 9 years of age. ${ }^{[3]}$

In 2014, the 9-valent HPV vaccine that adds five additional HPV genotype coverage to the original four HPV genotypes covered by the quadrivalent vaccine became available. ${ }^{[3]}$ The first indication listed in the current package insert for the 9valent vaccine is the prevention of "cervical, vulvar, vaginal and anal cancer". ${ }^{[4]}$ Although HPV vaccination prior to sexual acquisition of the virus is safe and effective in preventing HPV-associated cancers, 3-dose vaccine completion rates were only $39.7 \%$ for girls and $21.6 \%$ for boys in $2014 .^{[5,6]}$ If the HPV vaccination completion rates in the United States were raised to $80 \%$ by 2020 , some 53,000 cervical cancers could be prevented later in life for girls younger than 12 years of age. ${ }^{[6]}$

Despite the safety and efficacy of HPV vaccines, barriers to HPV vaccination include lack of parental awareness of vaccine benefits, perceived vaccine harm, concern of increased sexually activity of their children and lack of provider recommendations. ${ }^{[7-9]}$ Although Hispanics and African Americans have higher cervical cancer incidence and mortality, HPV knowledge and vaccination rates are lower compared to Caucasians. ${ }^{[2,10]}$ The low vaccination rates among minority groups are attributable to decreased access and utilization of health care, lack of insurance coverages, negative attitudes toward HPV vaccines and a lower likelihood of receiving provider recommendations. ${ }^{[1-13]}$

The combination of providers' discussion with young women and their recommendations for vaccination was associated with 93-fold higher likelihood of starting HPV vaccination, whereas strong recommendation alone was associated with a 4-fold higher likelihood of vaccination compared to weak recommendation. ${ }^{[14]}$ Nevertheless, it was reported that only $64 \%$ of girls' parents and $42 \%$ of boys' parents received recommendations for HPV vaccination. ${ }^{[15]}$ Another study reported that parents received weaker recommendations for HPV vaccination compared with other routine vaccines. ${ }^{[16]}$

Among the studies of healthcare providers, the low rates of their HPV vaccination recommendations were associated with providers' lack of knowledge, reluctance to discuss sex-related health issues with parents of children who may not be sexually active and providers' lack of confidence in their own ability to persuade parents to vaccinate. ${ }^{[8,17,18]}$ Less than half of the healthcare providers, including physicians, nurses, nurse practitioners and nurse midwives, did not know the recommended age for HPV vaccination or the recommended dosing intervals. ${ }^{[19]}$ Even though the primary indication for HPV vaccination is cancer prevention, more than half of family nurse practitioners did not know that HPV vaccines also prevent anal, vaginal, vulvar, or oropharyngeal cancers. ${ }^{[20]}$ Another study reported that patients of nurse practitioners or midwives were less likely to receive the complete HPV vaccine series, compared with patients of pediatricians or obstetricians. ${ }^{[21]}$

In the past, nurses have played a major role in improving childhood vaccinations against other infectious diseases through nurse-administered programs or by influencing parents. ${ }^{[22-24]}$ Equipped with updated HPV-vaccine knowledge, nurses can better educate and empower parents for timely HPV vaccinations. Various educational strategies in healthcare practice or community settings have shown to be effective in improving parental HPV vaccine knowledge and uptake. ${ }^{[25,26]}$ Although educating parents plays a key role in their decision-making process, the impact of the parentprovider relationship and strength of provider recommendation on HPV vaccination uptake or intent has not been fully examined. ${ }^{[12]}$

\section{Study aims}

The aims of this study were to: (a) explore parental knowledge, perceived HPV vaccine effectiveness and harm, provider recommendation for HPV vaccine and the parentprovider relationship; and (b) examine the predictors of HPV vaccine uptake or intent among parents of pre-adolescent and adolescent girls using path analysis.

Health Belief Model was used as a conceptual framework in this study for constructing the initial model to explore the various factors associated with parental decisions for having their children vaccinated or intent to vaccinate. Health Belief Model explains the various factors involved in preventive healthcare actions such as vaccinations. ${ }^{[27-29]}$ Some of the factors include the individual perceptions such as perceived vaccine benefits, perceived vaccine harm as a barrier, as well as perceived susceptibilities and severity of the diseases. Other factors in this model include demographic and socio-psychological factors of education or parent-provider relationship, as well as providers' recommendations representing cues for action.

\section{METHODS}

\subsection{Design and participants}

A cross-sectional descriptive study was conducted among parents of girls aged 9 to 18 years from September to November 2015 . 


\subsection{Data collection tools}

A study questionnaire included items from various published studies and demographic variables. Question items assessed parents' awareness of HPV vaccines, HPV knowledge and prior HPV vaccination recommendations by their children's primary care providers. ${ }^{[12,13,18]}$ Parents were asked about HPV vaccination history in a yes-no format; they were also asked about their intent to have their daughters vaccinated against HPV in the future on a 4-point response format ranging from 1 (definitely will not) to 4 (definitely will). ${ }^{[30,31]}$ Two items from the Caroline HPV Immunization Attitudes and Beliefs Scale (CHIAS) asked whether HPV vaccination is effective against cervical cancer and genital warts on a 4-point response format ranging from 1 (slightly effective) to 4 (extremely effective). ${ }^{[30]}$ Four items from CHIAS assessed the potentially harmful effects of HPV vaccines, such as vaccine side effects or the child becoming more sexually active on a 4-point response format ranging from 1 (strongly disagree) to 4 (strongly agree). Cronbach's alphas were 0.61 for the effectiveness factor and 0.69 for the harm factor. ${ }^{[30]}$

The Kim Alliance Scale-R (KAS-R) was used to assess parents' perceptions of therapeutic alliances with their children's primary care providers on a 4-point response format ranging from 1 (rarely) to 4 (always). ${ }^{[32]}$ The 16 -item KAS-R consists of four subscales: Integration subscale focusing on mutual respect, communication subscale assessing the quality of provider instructions, collaboration subscale evaluating the quality of cooperation to reach the shared goals and empowerment subscale evaluating the parent as an equal partner in the decision-making process. The internal consistency reliabilities were reported with Cronbach's alphas between 0.75 and 0.80 for the four subscales.

\subsection{Data collection procedures}

The study was reviewed and approved by the university institutional review board. Parents of girls aged 9 to 18 were asked to participate in the study while in the waiting rooms or examination rooms of two primary care clinics in central Texas. They were reminded that their participation in the study is entirely voluntary and they can withdraw from the study any time without any penalty. A written documentation of consent was waived since this study involved minimal risks to participants and completion of the questionnaire indicated their consent to participate in the study.

\subsection{Data analysis}

Means, standard deviations, frequencies and percentages were calculated to describe the demographic characteristics and study variables. In this study, the dependent variable was HPV vaccine uptake or intent, which was a combined result of the items regarding past HPV vaccination history and future intent to have their daughters vaccinated. A response of "yes" to the past HPV vaccination history item and response of "definitely will" for the future intent item were scored as positive HPV vaccine uptake or intent. Pearson's productmoment correlation procedures were performed to explore the relationships among the dependent variable and other variables, including parental HPV awareness and knowledge, perceived vaccine effectiveness and harm, report of provider recommendation for HPV vaccination and quality of parentprovider relationship, as well as demographic characteristics.

Path analyses using structural equation modeling with the initial model based on Health Belief Model were performed to explore predictors of HPV vaccine uptake or intent. Because of the small sample size, following goodness-of-fit indices were used in evaluating the models: $\chi^{2} / \mathrm{df}<2.0$, comparative fit index $(\mathrm{CFI})>0.90$, root mean square error of approximation (RMSEA) $<0.100 .{ }^{[33]}$ The best-fitting model with the most parsimonious significant paths was chosen as the final model. The SPSS version 22 and SPSS Amos version 23 statistical programs were used (IBM, Chicago, IL, USA). In this study, the level of significance was set at $p<.05$.

\section{RESULTS}

\subsection{Demographic characteristics}

A total of 60 parents from two primary care clinics completed the study questionnaire (see Table 1). The majority of parents were Caucasians (66.7\%) and college-educated (58.3\%). The mean ages of the parents and daughters were 41 and 13 years, respectively. The average duration of parent-provider relationship was 8.3 years, but only $63.3 \%$ of parents reported receiving recommendations for HPV vaccination from the providers. Although most of the parents were aware of HPV (95\%), only $45 \%$ of parents had vaccinated their daughters previously or would definitely vaccinate.

\subsection{Knowledge, vaccine effectiveness/harm and parent- provider relationship}

Most of the parents demonstrated good HPV knowledge by correctly identifying HPV-associated genital warts and cervical cancer (71.7\% and $80 \%$, respectively). Also, $70 \%$ of parents understood that HPV infections could present without any symptoms. The mean score for perceived HPV vaccine effectiveness was $2.4(\mathrm{SD} \pm 0.8)$, which is between "moderately effective" and "very effective". The mean score for perceived HPV vaccine harm was $2.2(\mathrm{SD} \pm 0.6)$, which is close to "somewhat disagree". The parent-provider relationship as measured by KAS-R was very good, with mean subscale scores ranging from 3.72 to 3.80 . Among the subscales, the mean score for empowerment subscale was 3.72 with a range 
of 2.5 to 4.0 , indicating high levels of parents perceiving themselves as equal partners in the decision-making process.

Table 1. Demographic characteristics $(\mathrm{N}=60)$

\begin{tabular}{ll}
\hline Variables & $\boldsymbol{n}(\%)$ \\
\hline Parent's age, mean (year), range & $41(28-61)$ \\
Parental gender & \\
Female & $57(95)$ \\
Male & $3(5.0)$ \\
Ethnicity & \\
White (Non-Hispanic) & $40(66.7)$ \\
Black & $2(3.3)$ \\
Hispanic & $10(16.7)$ \\
Asian/Pacific Islanders & $6(10.0)$ \\
Other & $2(3.3)$ \\
Parent's education & \\
High school or less & $6(10.0)$ \\
Some college & $19(31.7)$ \\
College graduate & $35(58.3)$ \\
Daughter's age, mean (year), range & $13(9-19)$ \\
Daughter's primary care provider & \\
Medical doctor & $57(95.0)$ \\
Nurse practitioner & $2(3.3)$ \\
Physician's assistant & $1(1.7)$ \\
Duration with primary care provider, year (range) & $8.3(1-18)$ \\
Provider recommendation for HPV vaccination & $38(63.3)$ \\
HPV awareness & $57(95.0)$ \\
Daughter vaccinated previously & $22(36.7)$ \\
Likelihood of HPV vaccination* & \\
Definitely will not & $6(10.0)$ \\
Probably will not & $17(28.3)$ \\
Probably will & $10(16.7)$ \\
Definitely will & $5(8.3)$ \\
\hline
\end{tabular}

Note. *Among the unvaccinated. Values are expressed as n (\%) unless otherwise indicated. Percentage may not add up to $100 \%$ because of missing data or rounding.

\subsection{Correlations}

Table 2 shows the results of bivariate Pearson's correlations among various variables. HPV vaccine uptake or intent had positive correlations with perceived HPV vaccine effectiveness $(r=0.35 ; p=.002)$ and provider recommendation for HPV vaccination $(r=0.39 ; p=.001)$, whereas it had a negative correlation with perceived HPV vaccine harm $(r=$ $-0.41 ; p<.001)$. Perceived HPV vaccine effectiveness had a negative correlation with perceived HPV vaccine harm $(r=$
$-0.42 ; p<.001)$, while it had a positive correlation with HPV knowledge $(r=0.37 ; p=.001)$. There was also a negative correlation between perceived HPV vaccine harm and HPV knowledge $(r=-0.22 ; p=.035)$.

\subsection{Predictors of HPV vaccine uptake or intent}

Figure 1 shows the initial model based on Health Belief Model. Model fit statistics showed this initial model did not meet the criteria for goodness of fit $\left(\chi^{2}=78.5, \mathrm{df}=35, p\right.$ $<.001, \chi^{2} / \mathrm{df}=2.24 ; \mathrm{CFI}=0.778 ;$ RMSEA $=0.145$ with 90\% Confidence Interval [CI], 0.102-0.188). Because of the poorly fitting initial model, an alternate model satisfying the criteria of goodness of fit was sought with statistically significant paths among the factors.

The final model with satisfactory goodness-of-fit indices is shown in Figure $2\left(\chi^{2}=58.3, \mathrm{df}=39, p=.024, \chi^{2} / \mathrm{df}=\right.$ 1.496 ; $\mathrm{CFI}=0.901$; RMSEA $=0.092$ with $90 \% \mathrm{CI}, 0.034$ $0.138)$. First, the paths showed that provider recommendation for HPV vaccination $(\beta=0.37 ; p<.001)$ and perceived HPV vaccine harm $(\beta=-0.48 ; p<.001)$ had statistically significant direct effects on HPV vaccine uptake or intent. The combination of provider recommendation for HPV vaccination, perceived HPV vaccine harm and demographic variables explained $41 \%$ of the variance in HPV vaccine uptake or intent $\left(R^{2}=0.41\right)$. Second, the paths also showed that perceived HPV vaccine effectiveness did not have a direct effect on HPV vaccine uptake or intent. However, it had a statistically significant direct effect on perceived HPV vaccine harm $(\beta=-0.56 ; p<.001)$. The combinations of perceived HPV vaccine effectiveness and demographic variables explained $32 \%$ of the variance in perceived HPV vaccine harm $\left(R^{2}=0.32\right)$. Third, the perceived HPV vaccine effectiveness was directly influenced by HPV knowledge $(\beta=0.39 ; p<$ $.001)$, empowerment in parent-provider relationships $(\beta=$ $0.30 ; p=.006)$, and parental college education $(\beta=0.23 ; p=$ .039). The combinations of these and demographic variables explained $29 \%$ of the variance in perceived HPV vaccine effectiveness $\left(R^{2}=0.29\right)$. Table 3 presents the standardized regression coefficients estimates for the final model.

Table 2. Correlations among variables $(\mathrm{N}=60)$

\begin{tabular}{llll}
\hline & HPV vaccine uptake or intent & $\begin{array}{l}\text { Perceived HPV vaccine } \\
\text { effectiveness }\end{array}$ & $\begin{array}{l}\text { Perceived HPV vaccine } \\
\text { harm }\end{array}$ \\
\hline Perceived HPV vaccine effectiveness & $0.35^{* *}$ & - & - \\
Perceived HPV vaccine harm & $-0.41^{* * *}$ & $-0.42^{* * *}$ & - \\
HPV knowledge & 0.13 & $0.37^{* *}$ & $-0.22^{*}$ \\
HPV awareness & 0.20 & 0.10 & -0.13 \\
Provider recommendation & $0.39^{* *}$ & 0.17 & -0.09 \\
Empowerment & 0.11 & 0.21 & -0.14 \\
College education & -0.09 & 0.22 & -0.15 \\
\hline
\end{tabular}

Note. ${ }^{*} p<.05 ; * * p<.01 ; * * * p<.001$ by Pearson's correlations. HPV: Human papillomavirus 


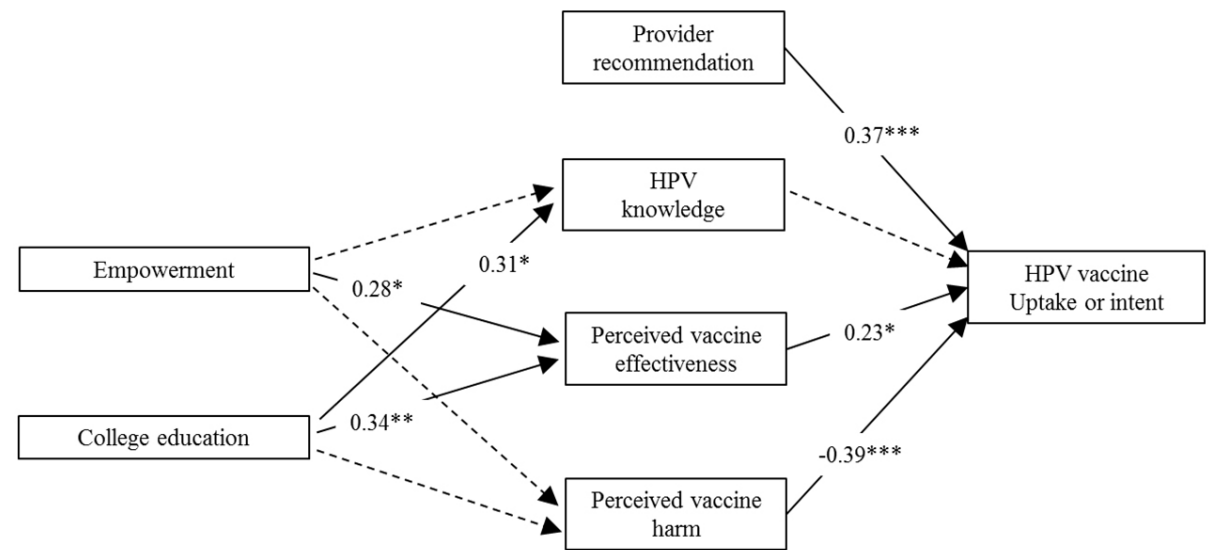

Figure 1. Initial model for HPV vaccine uptake or intent

Note. $* p<.05 ; * * p<.01 ; * * * p<.001 ;$ Model fit statistics: $\chi^{2}=78.5, d f=35, p<.001, \chi^{2} / d f=2.24$; Comparative fit index $($ CFI) $=$ 0.778; root mean square error of approximation (RMSEA) $=0.145$ (90\% Confidence Interval [CI], 0.102-0.188). Solid path arrows represent statistically significant paths; dotted path arrows represent non-significant paths.

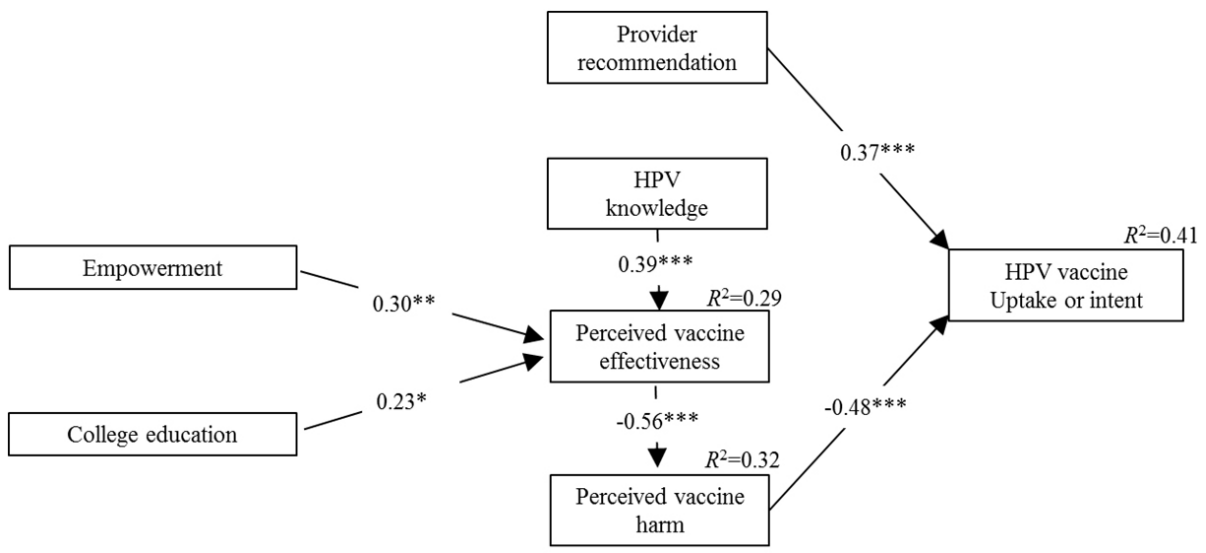

Figure 2. Final model for HPV vaccine uptake or intent

Note. ${ }^{*} p<.05 ; * * p<.01 ; * * *<<.001$; Final model fit statistics: $\chi^{2}=58.3, d f=39, p=.024, \chi^{2} / d f=1.496$; Comparative fit index $(C F I)=0.901$; root mean square error of approximation $(R M S E A)=0.092$ (90\% confidence interval [CI], 0.034-0.138). Solid path arrows represent statistically significant paths.

Table 3. Standardized regression coefficients estimate for the final model

\begin{tabular}{lll}
\hline Path & Coefficient estimate $(\boldsymbol{\beta})$ & $\boldsymbol{p}$ value \\
\hline Perceived HPV vaccine effectiveness $\leftarrow$ Empowerment & 0.30 & $.006^{* *}$ \\
Perceived HPV vaccine effectiveness $\leftarrow$ HPV knowledge & 0.39 & $<.001^{* * *}$ \\
Perceived HPV vaccine effectiveness $\leftarrow$ College education & 0.23 & $.039^{*}$ \\
Perceived HPV vaccine harm $\leftarrow$ Perceived HPV vaccine effectiveness & -0.56 & $<.001^{* * *}$ \\
HPV vaccine uptake or intent $\leftarrow$ Perceived HPV vaccine harm & -0.48 & $<.001^{* * *}$ \\
HPV vaccine uptake or intent $\leftarrow$ Provider recommendation & 0.37 & $<.001^{* * *}$ \\
\hline
\end{tabular}

Note. ${ }^{*} p<.05 ; * * p<.01 ; * * * p<.001$.

\section{Discussion}

Path analysis was used in this study to examine predictors of HPV vaccine uptake or intent among parents of preadolescent and adolescent girls. This allowed a diagrammatic

Published by Sciedu Press representation of the relationships among multiple variables with direct and indirect effects on HPV vaccine uptake or intent. The final model showed that provider recommendation had a significant direct effect on HPV vaccine uptake or 
intent. In contrast, parental empowerment, HPV knowledge and their college educational level had indirect effects on HPV vaccine uptake or intent.

Although provider recommendation had a significant direct effect on HPV vaccine uptake or intent, only $63.3 \%$ of parents reported that they had received provider recommendations, which is a major impediment to achieving high HPV vaccination rates. For HPV vaccine recommendation to parents, one useful communication strategy for healthcare providers is to focus on the cancer-preventive effects of HPV vaccination rather than the future sexual activities of their prepubescent children. ${ }^{[15,18]}$ It is important to note that the first listed indication in the package insert of the 9-valent HPV vaccine is the prevention of cancers. ${ }^{[4]}$ Other potential strategy for maximizing HPV vaccination may include mandatory HPV vaccinations for school entry, which will ensure timely vaccination of most children and minimize the morbidity and mortality associated with the preventable HPV-associated cancers. ${ }^{[34]}$

In this study, parental HPV knowledge and empowerment had positive direct effects on parental perception of HPV vaccine effectiveness. Together with parental empowerment fostering an equal partnership with providers, targeted education to improve parental HPV knowledge may convince them of the HPV vaccine effectiveness. This, in turn, may help them put the perceived HPV vaccine harm in proper perspective and allow them to make informed decisions regarding the timely HPV vaccination of their children. ${ }^{[16,35]}$

It was an interesting finding that perceived HPV vaccine effectiveness did not have a direct effect on HPV vaccine uptake or intent according to the path analysis. It is plausible that parents are more influenced by the misperception of harm from HPV vaccination than by long-term benefits. These parental misperceptions of HPV vaccination are consistent with previous study findings on barriers to HPV vaccination, such as parental knowledge gaps and fear of increased sexual activity following vaccination. ${ }^{[7]}$

\section{Limitations}

There are certain limitations to this study. First, the predictors for HPV vaccine uptake or intent should not be taken as cause-and-effect relationships, because this was not a randomized interventional study. Second, the small sample size of this study may not have sufficient power to detect other paths with small effect sizes. Third, this study was conducted in a single geographic location, which may limit generalizability of the study findings. Fourth, the study findings may not apply to parents of boys, because only parents of preadolescent and adolescent girls were studied. Finally, the study questionnaire assessing parental knowledge and perceived HPV vaccine effectiveness did not include items regarding various HPV-associated cancers oropharyngeal, anal, vulvar, penile, vaginal and rectal cancers. Future studies need to include parents of both genders, as well as question items that assess parents' knowledge regarding all HPV-associated cancers.

\section{Conclusions}

The current HPV vaccination rates among preadolescents and adolescents are suboptimal despite the guideline recommendations. Once young people get infected with HPV through sexual activities, the vaccine is no longer effective in preventing HPV-associated cancers such as cervical, oropharyngeal, anal, vulvar, penile, vaginal and rectal cancers. Optimal vaccination of children at the recommended age of 11-12 years will markedly reduce the burden of these cancers later in their lives. Since the nursing profession had made major contributions toward successful vaccinations that have resulted in near-elimination of many serious childhood infections in the past, the nurses can again play significant roles in maximizing timely HPV vaccinations. Recommendations related to HPV vaccination are changing rapidly, so it is critical for nurses to keep up with the most updated evidence and adhere to the current guideline recommendations. Because provider recommendation is one of the most important contributing factors for HPV vaccine uptake or intent, parental education and recommendations from nurses will help reduce the knowledge gaps and empower parents to make the timely decisions to vaccinate their children.

\section{CONFlicts of InTERest Disclosure}

The authors declare that there is no conflict of interest.

\section{REFERENCES}

[1] Chesson HW, Ekwueme DU, Saraiya M, et al. The estimated impact of human papillomavirus vaccine coverage on the lifetime cervical cancer burden among girls currently aged 12 years and younger in the United States. Sexually Transmitted Diseases. 2014; 41(11): 656-659.
PMid:25299411 https://doi.org/10.1097/OLQ.0000000000 000199

[2] Viens LJ, Henley SJ, Watson M, et al. Human papillomavirusassociated cancers - United States, 2008-2012. MMWR Morbidity Mortality Weekly Report. 2016; 65(26): 661-666. PMid:27387669 
https://doi.org/10.15585/mmwr.mm6526a1

[3] American College of Obstetricians and Gynecologists. Human papillomavirus vaccination. Committee Opinion No. 641. Obstetrics \& Gynecology. 2015; 126: e38-43.

[4] Merck Co, Inc. GARDASIL $® 9$ (Human Papillomavirus 9-valent vaccine, Recombinant). Highlights of prescribing information. 2015.

[5] Markowitz LE, Liu G, Hariri S, et al. Prevalence of HPV After introduction of the vaccination program in the United States. Pediatrics. 2016; 137(3): e20151968.

[6] Reagan-Steiner S, Yankey D, Jeyarajah J, et al. National, regional, state, and selected local area vaccination coverage among adolescents Aged 13-17 Years-United States, 2014. MMWR Morbidity Mortality Weekly Report. 2015; 64(29): 784-792. PMid:26225476 https://doi.org/10.15585/mmwr.mm6429a3

[7] Holman DM, Benard V, Roland KB, et al. Barriers to human papillomavirus vaccination among US adolescents: a systematic review of the literature. JAMA Pediatrics. 2014; 168(1): 76-82. PMid:24276343 https://doi.org/10.1001/jamapediatrics .2013 .2752

[8] Lindley MC, Jeyarajah J, Yankey D, et al. Comparing human papillomavirus vaccine knowledge and intentions among parents of boys and girls. Human Vaccines \& Immunotherapeutics. 2016; 12(6): 15191527. PMid:27003108 https://doi .org/10.1080/21645515.2 016.1157673

[9] Small SL, Sampselle CM, Martyn KK, et al. Modifiable influences on female HPV vaccine uptake at the clinic encounter level: A literature review. Journal of the American Association of Nurse Practitioners. 2014; 26(9): 519-525. PMid:24170346 https://doi.org/10.1 $002 / 2327-6924.12057$

[10] Galbraith KV, Lechuga J, Jenerette CM, et al. Parental acceptance and uptake of the HPV vaccine among African-Americans and Latinos in the United States: A literature review. Social Science \& Medicine. 2016; 159: 116-126. PMid:27180256 https: //doi.org/10.1016/j.socscimed.2016.04.028

[11] Jeudin P, Liveright E, Del Carmen MG, et al. Race, ethnicity, and income factors impacting human papillomavirus vaccination rates. Clinical Therapeutics. 2014; 36(1): 24-37. PMid:24417783 https://doi.org/10.1016/j.clinthera.2013.11.001

[12] Staras SA, Vadaparampil ST, Patel RP, et al. Parent perceptions important for HPV vaccine initiation among low income adolescent girls. Vaccine. 2014; 32(46): 6163-6169. PMid:25180815 https://doi.org/10.1016/j.vaccine. 2014.08.054

[13] Wisk LE, Allchin A, Witt WP. Disparities in human papillomavirus vaccine awareness among U.S. parents of preadolescents and adolescents. Sexually Transmitted Diseases. 2014; 41(2): 117-122. PMid:24413492 https://doi.org/10.1097/OLQ.0000000000 000086

[14] Rosenthal SL, Weiss TW, Zimet GD, et al. Predictors of HPV vaccine uptake among women aged 19-26: Importance of a physician's recommendation. Vaccine. 2011; 29(5): 890-895. PMid:20056186 https://doi.org/10.1016/j.vaccine.2009.12.063

[15] Stokley S, Jeyarajah J, Yankey D, et al. Human papillomavirus vaccination coverage among adolescents, 2007-2013, and postlicensure vaccine safety monitoring, 2006-2014-United States. MMWR Morbidity Mortality Weekly Report. 2014; 63(29): 620-624.

[16] Dempsey AF, Pyrzanowski J, Lockhart S, et al. Parents' perceptions of provider communication regarding adolescent vaccines. Human Vaccines \& Immunotherapeutics. 2016; 12(6): 14691475. PMid:27078515 https://doi .org/10.1080/21645515.2 016.1147636

[17] McRee AL, Gilkey MB, Dempsey AF. HPV vaccine hesitancy: Findings from a statewide survey of health care providers. Jour- nal of Pediatric Health Care. 2014; 28(6): 541-549. PMid:25017939 https://doi.org/10.1016/j.pedhc.2014.05.003

[18] Ylitalo KR, Lee H, Mehta NK. Health care provider recommendation, human papillomavirus vaccination, and race/ethnicity in the US National Immunization Survey. American Journal of Public Health 2013; 103(1): 164-169. PMid:22698055 https://doi.org/10.2 105/AJPH. 2011.300600

[19] Berenson AB, Rahman M, Hirth JM, et al. A brief educational intervention increases providers' human papillomavirus vaccine knowledge. Human Vaccines \& Immunotherapeutics. 2015; 11(6): 13311336. PMid:25945895 https://doi .org/10.1080/21645515.2 015.1022691

[20] Berkowitz Z, Malone M, Rodriguez J, et al. Providers' beliefs about the effectiveness of the HPV vaccine in preventing cancer and their recommended age groups for vaccination: Findings from a provider survey, 2012. Preventive Medicine. 2015; 81: 405-411. PMid:26598805 https://doi.org/10.1016/j.ypmed.2015.1 0.007

[21] Hirth JM, Tan A, Wilkinson GS, et al. Completion of the human papillomavirus vaccine series among insured females between 2006 and 2009. Cancer. 2012; 118(22): 5623-5629. PMid:22544681 https://doi.org/10.1002/cncr.27598

[22] Hill MC, Cox CL. Influencing factors in MMR immunisation decision making. British Journal of Nursing. 2013; 22(15): 893 898. PMid:24005660 https://doi.org/10.12968/bjon. 2013 .22 .15 .893

[23] Scott K, Batty ML. HPV vaccine uptake among Canadian youth and the role of the nurse practitioner. Journal of Community Health. 2016; 41(1): 197-205. PMid:26245727 https://doi .org/10.1007/s1 0900-015-0069-2

[24] Zelman M, Sanford C, Neatby A, et al. Implementation of a universal rotavirus vaccination program: Comparison of two delivery systems. BMC Public Health. 2014; 14: 908. PMid:25182067 https://doi.org/10.1186/1471-2458-14-908

[25] Fu LY, Bonhomme LA, Cooper SC, et al. Educational interventions to increase HPV vaccination acceptance: A systematic review. Vaccine. 2014; 32(17): 1901-1920. PMid:24530401 https : //doi.org/10.1016/j.vaccine.2014.01.091

[26] Niccolai LM, Hansen CE. Practice- and community-based interventions to increase human papillomavirus vaccine coverage: A systematic review. JAMA Pediatrics. 2015; 169(7): 686-692. PMid:26010507 https://doi.org/10.1001/jamapediatrics .2015 .0310

[27] Gerend MA, Shepherd JE. Predicting human papillomavirus vaccine uptake in young adult women: Comparing the health belief model and theory of planned behavior. Annals of Behavioral Medicine. 2012; 44(2): 171-180. PMid:22547155 https://doi.org/10.1 007/s12160-012-9366-5

[28] Guvenc G, Seven M, Akyuz A. Health Belief Model Scale for Human Papilloma Virus and its vaccination: Adaptation and psychometric testing. Journal of Pediatric and Adolescent Gynecology. 2016; 29(3): 252-258. PMid:26409648 https://doi.org/10.1016/j. jpag. 2015.09.007

[29] Rosenstock IM, Strecher VJ, Becker MH. Social learning theory and the Health Belief Model. Health Education Quarterly. 1988; 15(2): 175-183. https://doi.org/10.1177/109019818801500203

[30] McRee AL, Brewer NT, Reiter PL, et al. The Carolina HPV immunization attitudes and beliefs scale (CHIAS): scale development and associations with intentions to vaccinate. Sexually Transmitted Diseases. 2010; 37(4): 234-239. PMid:19940807

[31] Reiter PL, Brewer NT, Gottlieb SL, et al. Parents' health beliefs and HPV vaccination of their adolescent daughters. Social Sci- 
ence \& Medicine. 2009; 69(3): 475-480. PMid:19540642 https : //doi.org/10.1016/j.socscimed.2009.05.024

[32] Kim SC, Kim S, Boren D. The quality of therapeutic alliance between patient and provider predicts general satisfaction. Military Medicine 2008; 173(1): 85-90. https://doi.org/10.7205/MILMED.173 .1 .85

[33] Hooper D, Coughlan J, Mullen M. Structural equation modeling: Guidelines for determining model fit. Electronic Journal of Business Research Methods. 2008; 6(1): 53-60.
[34] Gilkey MB, McRee AL. Provider communication about HPV vaccination: A systematic review. Human Vaccines \& Immunotherapeutics. 2016; 12(6): 1454-1468. PMid:26838681 https://doi.org/10.1 $080 / 21645515.2015 .1129090$

[35] Wegwarth O, Kurzenhauser-Carstens S, Gigerenzer G. Overcoming the knowledge-behavior gap: The effect of evidence-based HPV vaccination leaflets on understanding, intention, and actual vaccination decision. Vaccine. 2014; 32(12): 1388-1393. PMid:24486360 https://doi.org/10.1016/j.vaccine.2013.12.038 